\title{
Docencia en Aulas Multigrado: Claves para la Calidad Educativa y el Desarrollo Profesional Docente
}

\author{
Multilateral Education: Key Words for Educational Quality and \\ Professional Development
}

\author{
Luis Felipe de la Vega* \\ Universidad Cardenal Silva Henríquez, Chile
}

\begin{abstract}
Este trabajo busca describir y analizar desafíos que se presentan a la enseñanza en aulas multigrado y, a partir de allí, sugerir claves relevantes para la calidad educativa y el desarrollo profesional de los docentes que se desempeñan en esos contextos, las que se inspiran en el paradigma de educación inclusiva. A partir de la observación de clases y de la realización de entrevistas a catorce docentes encargados de ese tipo de instituciones, se vislumbró un énfasis en un tipo de enseñanza individualizada, tensionada por la búsqueda de diseños de clase que permitan cubrir el currículum, considerando la gran heterogeneidad de niveles de enseñanza que cursan los estudiantes que conviven en una misma aula. Estas dinámicas pedagógicas ocurren en un escenario marcado por la debilidad de políticas y apoyos contextualizados por parte del sistema educativo. Las claves para el desarrollo profesional que se presentan en base a los resultados buscan enfatizar los aportes que puede entregar el paradigma de la educación inclusiva en el contexto estudiado, debido al énfasis que ésta hace respecto del rol de la pedagogía en la entrega de apoyos, el diseño de experiencias de aprendizaje que consideren las características y especificidades de los alumnos y alumnas y que propicien y promuevan el desarrollo de su autodeterminación.
\end{abstract}

Descriptores: Aulas multigrado; Calidad educativa; Desarrollo profesional docente; Educación rural; Educación Inclusiva.

This work seeks to describe and analyze challenges for teaching in multigrade classrooms and, from there, to suggest relevant keys for the educational quality and professional development of teachers working in these contexts, that are inspired by the inclusive education paradigm. From the observation of classes and conducting interviews with fourteen teachers in charge of such institutions, an emphasis was identified on a type of individualized teaching, stressed by the search for class designs that allow covering the curriculum, considering the large heterogeneity of levels of education that students who live in the same classroom. These pedagogical dynamics occur in a scenario characterized by the weakness of contextualized policies and support from the educational system. The keys to professional development that are presented based on the results seek to emphasize the contributions that the inclusive education paradigm can provide in this context, due to the emphasis that it makes regarding the role of pedagogy in the delivery of supports, the design of experiences that take into account the characteristics and specificities of students and that encourage and promote the development of their self-determination

Keywords: Multigrade classrooms; Educational quality; Professional teacher development; Rural education; Inclusive Education. 


\section{La calidad educativa y la relevancia que en ella tienen los procesos de enseñanza-aprendizaje}

La discusión sobre la calidad ha sido un patrón permanente en diferentes sistemas educativos en las últimas décadas (Tiana, 2009). Según Coll (2009), analizar la calidad de la educación implica hacerse tres preguntas fundamentales: cuáles son los objetivos y finalidades de la educación; qué características debiera tener el currículum escolar para responder a las necesidades de los estudiantes y la sociedad y de qué forma los sistemas educativos responden a exigencias educativas y necesidades de aprendizaje cada vez más diversas. Además de reconocer la integralidad de dimensiones que componen la calidad educativa, se ha generado cierto consenso en que el objetivo de la calidad es de naturaleza sistémica, y que, por lo tanto, no depende únicamente del trabajo del docente (Mourshed, Chijioke y Barber, 2010).

Las últimas décadas han estado marcadas además por requerimientos y acciones orientadas a que los sistemas educativos propicien una educación de calidad para cada estudiante que participa de los procesos formativos, considerando el paradigma de la inclusión educativa. Para ello, entre otros atributos, se requiere que los sistemas aseguren la utilización de criterios de inclusión fundamentales, tales como son los de presencia, aprendizaje y participación (Ainscow, Booth y Dyson, 2006; Arnaiz, Escarbajal y Caballero, 2017).

La calidad educativa se expresa especialmente al interior del aula y las prácticas pedagógicas son un proceso fundamental en ese escenario (Martinic y Vergara, 2007; OEDC, 2010). En línea con lo anterior, buena parte de los estudios sobre mejora escolar ha ido privilegiando crecientemente el análisis de los elementos propios de la dinámica interior del aula, y el tipo de prácticas e interacciones que los docentes promueven (Godoy et al., 2016; Hopkins y Reynolds, 2002).

Pese al consenso existente sobre su relevancia, la evidencia empírica ha dado cuenta de importantes debilidades en la implementación del vínculo que se establece entre el docente y los estudiantes en los procesos de enseñanza (Valenzuela et al., 2009), lo que ha permitido identificar variables y criterios favorecen la calidad educativa en estos espacios (Scheerens, Luyten y Steen, 2005). Sin embargo, gran parte de esta investigación se ha realizado en establecimientos y clases "regulares", donde el docente interactúa con estudiantes de un mismo nivel, en un contexto institucional caracterizado por la división de funciones y la existencia de equipos técnicos. Pero, ¿qué ocurre en otro tipo de contextos de enseñanza y aprendizaje? El presente artículo aborda el estudio de la calidad educativa en un contexto de enseñanza multigrado.

\section{Desafíos para la calidad educativa en escuelas multigrado}

Las escuelas multigrado son instituciones educativas pequeñas, que trabajan con un número reducido de estudiantes, los que conviven en una misma sala, pero necesariamente coinciden en el grado que están cursando. Normalmente, esta condición está estrechamente asociada a una determinada situación geográfica, social o cultural, que genera una baja concentración de estudiantes y ciertas particularidades idiosincráticas. El docente a cargo de la institución normalmente asume el proceso formativo de todo el 
grupo y además otras funciones de administración del establecimiento (Boix, 2014; Boix y Bustos, 2014; Hamodi y Aragués, 2013).

Por las características que se describirán a continuación, se trata de un tipo de establecimiento que ha estado estructuralmente demandado por la necesidad de ser inclusivo. Por ello, se vuelve un objeto relevante de investigación para el análisis de la calidad educativa desde una perspectiva inclusiva.

Los sistemas educativos se organizan normalmente segmentando a los estudiantes según niveles que están asociados a una determinada edad e impartiendo un currículum que se organiza bajo esa lógica. Esta estructura no se ajusta completamente a las escuelas multigrado, lo que las convierte en espacios particularmente desafiantes para la enseñanza y el aprendizaje (Clarke y Stevens, 2009).

¿Qué ocurre con la calidad educativa en este tipo de establecimientos, en el contexto descrito? De acuerdo a Schwartz, Stiefel y Witwall (2016) y Galaz (2018) el aprendizaje pudiera verse favorecido debido al mayor grado de cercanía y pertenencia que se genera en ese espacio. Bustos (2010) agrega que la multigraduación propicia la realización de un monitoreo entre los propios estudiantes, hecho que fomenta que estos se ayuden mutuamente, aportando al aprendizaje entre pares.

En la vereda opuesta, un conjunto de la literatura reconoce un conjunto de desafíos asociados a la enseñanza en estas instituciones. Un ejemplo de ello es la necesidad de contextualizar el currículum nacional en un marco de constreñimientos de tiempo, recursos y espacios (Ávila, 2017; Downes y Roberts, 2016;UNESCO, 1989). Según Boix y Bustos (2014), la combinación entre la convivencia de estudiantes de diferentes edades y la existencia de un número individual o bajo de docentes, afectan en el tiempo disponible para la enseñanza y por ende, son altamente demandantes para el diseño pedagógico. Además son reconocidas las dificultades existentes para utilizar recursos disponibles para el resto del sistema educativo y problemas para acceder a internet o a TIC.

En definitiva, las escuelas rurales deben enfrentar permanentes "problemas de adaptación" (Fullan, 2005), debido a que pocas veces existen productos o respuestas hechas para considerar (Wildy y Clarke, 2011). Asimismo, la práctica pedagógica en escuelas multigrado enfrenta una serie de desafíos particulares, que requieren, entre otras, de habilidades específicas para identificar las necesidades individuales de aprendizaje de los estudiantes, considerando que no existe la homogeneidad por edad o grado (Centre for Education Statistics and Evaluation, 2013).

El problema es que los docentes de estas escuelas generalmente no han recibido formación específica para el trabajo en estos contextos, ni en la formación inicial ni continua (Clarke y Stevens, 2009; Downes y Roberts, 2016). Así, al trasladarse o desempeñarse como docente en una escuela rural o aislada, los profesores experimentan un conjunto de fenómenos para los que no han sido preparados profesionalmente: comprender a la comunidad y su expectativa sobre la educación, trabajar y adaptarse a grupos curso heterogéneos, establecer relaciones personales y profesionales en este entorno, manejar su visibilidad dentro de la comunidad, o sobrellevar una alta cercanía con sus colegas, cuando estos existen (Downes y Roberts, 2016).

En este contexto son fundamentales las acciones que pueden favorecer el desarrollo profesional docente. Procesos como las redes de colaboración entre docentes de diferentes 
escuelas o la inducción profesional pueden ayudar a suplir las brechas entre la formación inicial y la experiencia de la enseñanza cotidiana (Panizzon, 2011; Wildy y Clarke, 2011).

Las particularidades de los procesos pedagógicos en aulas multigrado presentan desafíos a los y las docentes que guardan una importante convergencia con la visión y propuestas del paradigma inclusivo. De acuerdo a la Agencia Europea para el Desarrollo de la Educación del Alumnado con Necesidades Educativas Especiales (2012) una educación de calidad en función de las necesidades y características de sus estudiantes, requiere de un desarrollo profesional que considere la reflexión sobre su propia práctica, la consideración por el bienestar del estudiantado, la valoración de la diversidad, la diversificación de prácticas pedagógicas y la colaboración.

Como puede observarse, dentro del marco de particulares desafíos para la calidad educativa en escuelas multigrado, la labor del docente tiene particular relevancia. Por esta razón, este estudio se pregunta por factores relevantes de considerar en la calidad de una clase en un contexto multigrado, de manera de identificar desafíos para el desarrollo profesional de los docentes que se desenvuelven allí.

Teniendo en consideración los factores relevados por la literatura en relación a la enseñanza en escuelas multigrado, el presente trabajo da cuenta de un estudio que busca identificar desafíos para la enseñanza en escuelas multigrado chilenas, de manera de, extraer desde allí claves que inciden en la calidad educativa en dichos contextos e identificar ámbitos relevantes para el desarrollo profesional docente.

\section{Metodología}

Para abordar el objeto de estudio, se utilizó un diseño mixto concurrente de triangulación (Creswell y Plano, 2007) que consideró a los docentes encargados de catorce establecimientos educacionales chilenos que imparten enseñanza multigrado en diversas regiones de Chile. El cuadro 1 entrega una caracterización de los establecimientos en que estos docentes se desempeñan.

\section{Instrumentos de producción de información}

El primer proceso de producción de información utilizado fue la observación de clases de los docentes encargados de estos establecimientos durante una visita hecha al establecimiento. La observación se realizó considerando una pauta semiestructurada, cuyo diseño fue organizado en dimensiones que enmarcaban ámbitos en que el/ la observador /a registraba sus observaciones de manera abierta. El instrumento fue aplicado in situ.

Se llevaron a cabo catorce procesos de observación de clases para cubrir el total de los casos mencionados. Las clases fueron observadas durante la jornada de visita al establecimiento educacional y se extendieron por alrededor de tres horas, con una variabilidad que dependió del horario de clases. La asignatura o asignaturas observadas en cada caso fueron definidas por cada docente. El proceso de observación fue realizado por el/la investigador/a que se encargó de la respectiva institución. Participó un total de seis observadores.

La pauta de observación fue diseñada para la realización de este estudio en particular, y tuvo como objetivo 1) describir el rol que asumen los distintos participantes de la clase y 2) dar cuenta de la dinámica que se desarrolla en la implementación de los procesos de enseñanza y aprendizaje. De esta forma, se buscó contar con una panorámica completa de 
lo que ocurre al interior de las clases que son objeto de estudio. Las dimensiones y ámbitos observados fueron los siguientes:

Cuadro 1. Participantes de la investigación

\begin{tabular}{|c|c|c|c|c|}
\hline $\mathrm{N}^{\circ}$ & REGIÓN & DEPENDENCIA & TIPO ESCUELA & AISLAMIENTO \\
\hline 1 & $\mathrm{RM}$ & Municipal & $\begin{array}{l}\text { Director(a) y al menos un } \\
\text { docente visitante }\end{array}$ & Leve \\
\hline 2 & $\mathrm{RM}$ & Municipal & $\begin{array}{l}\text { Director(a) y al menos un } \\
\text { docente visitante }\end{array}$ & Leve \\
\hline 3 & IV & Municipal & Unidocente & Leve \\
\hline 4 & $\mathrm{X}$ & Municipal & Unidocente & Leve \\
\hline 5 & IX & Municipal & $\begin{array}{l}\text { Director(a) y al menos un } \\
\text { docente visitante }\end{array}$ & Leve \\
\hline 6 & IV & $\begin{array}{l}\text { Particular } \\
\text { subvencionado }\end{array}$ & Unidocente & Intermedio \\
\hline 7 & IX & $\begin{array}{l}\text { SLEP Costa } \\
\text { Araucanía }\end{array}$ & $\begin{array}{l}\text { Director (a) y al menos un } \\
\text { docente visitante }\end{array}$ & Intermedio \\
\hline 8 & $\mathrm{X}$ & Municipal & $\begin{array}{l}\text { Director(a) y al menos un } \\
\text { docente visitante }\end{array}$ & Intermedio \\
\hline 9 & IV & Municipal & Unidocente & Agudo \\
\hline 10 & $\mathrm{X}$ & Municipal & Unidocente & Agudo \\
\hline 11 & $\mathrm{X}$ & Municipal & Unidocente & Agudo \\
\hline 12 & IX & $\begin{array}{l}\text { Particular } \\
\text { subvencionado }\end{array}$ & Unidocente & Sin aislamiento \\
\hline 13 & IX & $\begin{array}{l}\text { Particular } \\
\text { subvencionado }\end{array}$ & $\begin{array}{l}\text { Director(a) y al menos un } \\
\text { docente visitante }\end{array}$ & Agudo \\
\hline 14 & IX & Municipal & Unidocente & Intermedio \\
\hline
\end{tabular}

Fuente: Elaboración propia.

Dimensión 1. Relaciones entre los involucrados en la clase. Esta dimensión describió fenómenos asociados al rol que asumieron los participantes del proceso de enseñanzaaprendizaje en las clases observadas. Los ámbitos de observación fueron: a) disposición espacial de la sala; b) rol del docente en la clase; c) rol de los estudiantes en la clase; d) relaciones/ interacciones entre estudiantes.

Dimensión 2. Procesos de enseñanza-aprendizaje. Esta dimensión consideró aquellas prácticas, dinámicas y recursos que se identificaron en los procesos de enseñanza y aprendizaje que fueron observados. Los ámbitos de observación fueron: e) prácticas de enseñanza y actividades desarrolladas en la clase; f) Prácticas de monitoreo y evaluación utilizadas en la clase; g) vínculos entre contenidos de la clase y entorno/ vida cotidiana; h) recursos pedagógicos utilizados; i) clima de aula.

El segundo proceso de producción de información fue la realización de entrevistas semiestructuradas a los catorce docentes encargados de estos establecimientos, en las que se buscó reconocer su percepción respecto de la calidad educativa en contexto multigrado y factores que caracterizan y afectan el proceso de enseñanza y aprendizaje. Además, se indagó en las oportunidades, necesidades y evaluación de experiencias referidas al desarrollo profesional docente. Las entrevistas fueron complementadas con anotaciones realizadas por los/as investigadores/as que emergieron de conversaciones informales realizadas durante la visita al establecimiento. 


\section{Estrategias de análisis de la información}

Previo a la realización del trabajo de campo se realizó un pretest de su aplicación y una capacitación de cada investigador/a participante, para asegurar la calidad del registro y codificación de los observadores.

Luego, con los datos definitivos ya producidos y sobre la base de la descripción realizada en cada pauta de observación, el equipo de investigación -compuesto por cuatro personastrabajó de manera conjunta en la codificación y análisis de los datos, de manera de cautelar la calibración el análisis de los diferentes docentes observados. Para ello, a partir de los registros de las observaciones de clase, se realizó un primer ejercicio de codificación abierta en cada ámbito de observación y dimensión. Luego, estos episodios fueron categorizados, de manera de identificar patrones y diferencias entre los casos.

Las entrevistas fueron objeto de codificación abierta y axial, siguiendo los procesos definidos por la Grounded Theory (Strauss y Corbin, 2002).

Posteriormente, se realizó una integración de los resultados de ambos análisis, de manera de responder a la pregunta de investigación. Así, una vez habiendo descrito las características de la docencia multigrado, se buscó reconocer claves para la calidad educativa y desafíos para el desarrollo profesional en este contexto.

\section{Resultados}

\section{1. ¿Qué características tienen las clases en las escuelas multigrado que fueron observadas?}

En primer lugar, se hará una descripción de las principales características que se identificaron en las clases que fueron observadas, considerando las categorías descritas. Se iniciará con la descripción de la organización del espacio físico y el vínculo entre los involucrados.

La organización del espacio en las aulas en que se observaron clases no fue completamente homogénea. Un mayor número de las salas organizaban las mesas agrupando a estudiantes que están en el mismo nivel de enseñanza o en niveles cercanos. Sin embargo, otras salas ofrecían mayor libertad para la agrupación de los estudiantes. En algunas aulas los estudiantes quedaban en posición de mirar al docente, mientras otras privilegiaban reunir subgrupos de estudiantes, de manera de asegurar que puedan trabajar de forma colectiva. Se identificó un caso en que se habían dispuesto muebles para dividir la sala en dos espacios, de manera de favorecer la división de dos grupos de estudiantes, según el nivel de enseñanza que cursan.

Gran parte de las salas de clases observadas eran multifuncionales o contaban con múltiples elementos o recursos. En concreto, un grupo de salas de clases disponían de bibliotecas de aula o de espacios para guardar y disponer de libros o material bibliográfico. En otras, además este espacio es utilizado para guardar otros recursos pedagógicos, como mapas, proyectores o instrumentos. En algunos casos la sala de clases comparte espacio con otras roles dentro de la escuela, por ejemplo, la oficina del docente.

Como puede observarse, el espacio físico de clases genera desafíos de planificación por parte del docente, quien debe definir cómo deben participar o agruparse los estudiantes de diferente grado y a la vez resolver el problema de espacio disponible para los recursos de 
aprendizaje. Ambos elementos, ciertamente tendrán implicancia en el proceso de enseñanza-aprendizaje.

A continuación se describirán los resultados obtenidos de la observación, en relación a la primera de las dimensiones de análisis, referida al rol de los involucrados en proceso de enseñanza-aprendizaje.

\section{Rol general del docente en la sala de clases}

El cuadro 2 evidencia que en las clases observadas se identifican ciertos patrones respecto del rol que asume el docente en la clase. La mayor parte de ellas dispusieron de un momento de presentación de contenidos, relativamente corto en cuanto a su extensión, seguido por un momento de trabajo autónomo, de mayor extensión y con mayor frecuencia grupal, en que los estudiantes desarrollaban una actividad solicitada por el docente.

El primero de los momentos descritos fue similar al de docencia frontal que podría ocurrir en cualquier escuela. El segundo momento dispone de algunas particularidades. En primer lugar, la recurrencia de este tipo de dinámica hace inferir que es parte de la rutina cotidiana de las escuelas que este trabajo aplicado se haga permanentemente. En segundo lugar, la manera en que se organiza el trabajo aplicado tiene algunas características específicas.

Durante ese momento, los docentes estuvieron permanentemente visitando las mesas de los estudiantes o grupos, desarrollando un monitoreo y apoyo de manera permanente y ocupando en él gran parte del tiempo que se extendió la clase. Este proceso, se observó un espacio de enseñanza individualizada del docente, más allá del monitoreo de la actividad.

Por su parte, las actividades son organizadas por el docente siguiendo dos patrones. El primero fue reunir a estudiantes según el nivel de enseñanza que cursaban (solo un nivel o niveles aledaños) y solicitarles la realización de la actividad, con diferencias de dificultad, las que podían ser desde la cantidad de tareas que había que realizar, hasta elaborar procesos más complejos que los más pequeños. El segundo patrón observado era que todos realizaran la misma actividad, independiente del nivel que estén cursando. En algunos de estos casos, se identifica que el docente dedica mayor tiempo al estudiante menor del grupo curso o a algún estudiante que tenga alguna necesidad educativa especial.

Los patrones de uso del tiempo del docente en la clase describen la necesidad de generar instancias particularizadas para el desarrollo del aprendizaje del estudiante. La lógica frontal de clases demuestra claramente sus límites en este contexto, requiriendo que el docente busque opciones que le permitan identificar y responder con mayor especificidad a las necesidades de aprendizaje de sus estudiantes. 
Cuadro 2. Descripción de clases observadas. Dimensión involucrados en proceso de enseñanza- aprendizaje

\begin{tabular}{|c|c|c|c|c|c|c|c|c|c|c|c|c|c|c|c|}
\hline & & EsC & EsC & EsC & EsC & EsC & Esc & EsC & Esc & Esc & EsC & EsC & EsC & ESC & EsC \\
\hline & & 1 & 2 & 3 & 4 & 5 & 6 & 7 & & & 10 & 11 & 12 & 13 & 14 \\
\hline \multirow{7}{*}{$\begin{array}{l}\text { Rol del } \\
\text { docente }\end{array}$} & $\begin{array}{l}\text { Explicación actividades a realizar } \\
\text { y guía a estudiantes en resolución } \\
\text { de actividades }\end{array}$ & $\mathrm{x}$ & $\mathrm{x}$ & & & $\mathrm{x}$ & $\mathrm{x}$ & $\mathrm{X}$ & $\mathrm{x}$ & $\mathrm{x}$ & & & & & $\mathrm{x}$ \\
\hline & $\begin{array}{l}\text { Guía individualmente a } \\
\text { estudiantes en resolución de } \\
\text { actividades }\end{array}$ & & & $\mathrm{x}$ & $\mathrm{x}$ & & & & & & $\mathrm{x}$ & $\mathrm{X}$ & $\mathrm{x}$ & $\mathrm{x}$ & $\mathrm{x}$ \\
\hline & $\begin{array}{l}\text { Trabaja con una asistente de aula, } \\
\text { con quien se dividen grupos. }\end{array}$ & & & $\mathrm{x}$ & & & & & $\mathrm{x}$ & & & & & $\mathrm{x}$ & $\mathrm{x}$ \\
\hline & $\begin{array}{l}\text { Prioriza estudiante de } 1^{\circ} \text { básico/ } \\
\text { más pequeños }\end{array}$ & & & & $\mathrm{X}$ & & & & & & & & $\mathrm{X}$ & & \\
\hline & $\begin{array}{l}\text { Propicia aporte de alumnos } \\
\text { mayores a menores. }\end{array}$ & & & & & $\mathrm{x}$ & & & & & & & & & \\
\hline & Realiza evaluación de aprendizajes. & & & & & & $\mathrm{x}$ & & & & & & & & \\
\hline & $\begin{array}{l}\text { Reúne a estudiantes para } \\
\text { organizar resultados en conjunto. }\end{array}$ & & & & & & $\mathrm{X}$ & $\mathrm{X}$ & & & & & & & \\
\hline \multirow{8}{*}{$\begin{array}{l}\text { Rol de los } \\
\text { estudiantes }\end{array}$} & $\begin{array}{l}\text { Escuchan explicación del profesor } \\
\text { y responden preguntas. }\end{array}$ & $\mathrm{x}$ & $\mathrm{x}$ & & & $\mathrm{x}$ & $\mathrm{x}$ & $\mathrm{X}$ & $\mathrm{x}$ & $\mathrm{x}$ & & & & & \\
\hline & Trabajan individualmente & $\mathrm{X}$ & $\mathrm{X}$ & $\mathrm{X}$ & $\mathrm{X}$ & $\mathrm{X}$ & $\mathrm{X}$ & $\mathrm{X}$ & $\mathrm{X}$ & $\mathrm{X}$ & $\mathrm{x}$ & $\mathrm{X}$ & $\mathrm{X}$ & $\mathrm{x}$ & $\mathrm{X}$ \\
\hline & Hacen preguntas al docente & & & $\mathrm{x}$ & $\mathrm{x}$ & & & & & & $\mathrm{x}$ & $\mathrm{x}$ & $\mathrm{x}$ & $\mathrm{x}$ & $\mathrm{X}$ \\
\hline & $\begin{array}{l}\text { Participan colectivamente del } \\
\text { cierre }\end{array}$ & & & & & $\mathrm{x}$ & & $\mathrm{X}$ & & & & & & & \\
\hline & Trabajan grupalmente & & & & & & $\mathrm{X}$ & & & & & & & & \\
\hline & Presentan su trabajo al curso & & & & & & & $\mathrm{X}$ & $\mathrm{X}$ & $\mathrm{x}$ & & & & & \\
\hline & Trabajan organizados por nivel & & & & & & & & & & $\mathrm{x}$ & & $\mathrm{X}$ & $\mathrm{x}$ & \\
\hline & Trabajan organizados por sexo & & & & & & & & & & & & & & $\mathrm{x}$ \\
\hline
\end{tabular}




\begin{tabular}{|c|c|c|c|c|c|c|c|c|c|c|c|c|c|c|c|}
\hline \multirow{5}{*}{$\begin{array}{l}\text { Relaciones/ } \\
\text { interaccione } \\
\text { s entre } \\
\text { estudiantes }\end{array}$} & $\begin{array}{l}\text { Estudiantes trabajan } \\
\text { individualmente }\end{array}$ & $\mathrm{X}$ & $\mathrm{X}$ & $\mathrm{X}$ & $\mathrm{X}$ & $\mathrm{X}$ & $\mathrm{X}$ & $\mathrm{X}$ & $\mathrm{X}$ & $\mathrm{X}$ & $\mathrm{X}$ & $\mathrm{X}$ & $\mathrm{X}$ & $\mathrm{X}$ & $\mathrm{X}$ \\
\hline & Estudiantes se ayudan entre sí & & $\mathrm{X}$ & & & & & $\mathrm{X}$ & & & & & & $\mathrm{X}$ & $\mathrm{X}$ \\
\hline & $\begin{array}{l}\text { Estudiante(s) mayor (es) apoya }(\mathrm{n}) \\
\text { a compañero }(\mathrm{s}) \text { menores }\end{array}$ & & & & & $\mathrm{X}$ & & & & & & & & $\mathrm{X}$ & \\
\hline & $\begin{array}{l}\text { Trabajo colectivo entre } \\
\text { estudiantes }\end{array}$ & & & & & & $\mathrm{X}$ & & $\mathrm{X}$ & & & & & & \\
\hline & $\begin{array}{l}\text { Estudiantes apoyan a estudiante } \\
\text { con NEE }\end{array}$ & & & & & & & & & & & & & $\mathrm{X}$ & \\
\hline
\end{tabular}

Fuente: Elaboración propia. 


\subsection{Rol de los estudiantes en la sala de clases}

Debido a las características de la clase que propone el docente, los estudiantes pasaron la mayor parte del tiempo con un rol activo, debido a que estaban trabajando en la actividad solicitada por el docente.

Se observaron dos maneras de trabajar en las actividades. La primera fue de forma individual, en que el estudiante realiza su tarea en silencio. En estos casos se observa que los alumnos están concentrados en la realización de esta actividad y que hay poco intercambio entre los estudiantes. La segunda forma de realización de las actividades fue grupal, en conjuntos de estudiantes definidos por el docente y que con mayor regularidad, se organizan por nivel de enseñanza.

Un período de la clase en que los estudiantes tuvieron un rol más pasivo fue aquel en que el docente presentó contenidos generales al curso.

\subsection{Relaciones e interacciones entre los estudiantes}

Las relaciones entre los alumnos fueron por lo general cercanas y respetuosas, sin que se observaran grandes episodios de disrupción o problemas relacionales de relevancia. Pese a ello, la interacción entre los estudiantes fue baja, debido a que estaban realizando actividades o guías individuales, o bien se acotó al vínculo con la pareja o grupo en que se realiza la actividad.

Solo en algunas clases se observaron interacciones destinadas a la colaboración entre los estudiantes, considerando particularmente el escenario de apoyo de un estudiante a otro que tenía alguna dificultad y que pudo o no haberse solicitado explícitamente.

El fenómeno recién descrito da cuenta de que la particularización de la estrategias de enseñanza genera desafíos en relación a evitar el aislamiento entre los estudiantes, en un escenario que además está marcado por un bajo número de alumnos/as.

Frente a esta situación se identificó uno tipo de interacción de colaboración, la que ocurrió en un grupo minoritario de las clases. Esta fue la presencia evidente de un estudiante mayor como líder dentro de la clase. Este estudiante es requerido por el resto de los estudiantes, para la validación de actividades realizadas o para solicitar su opinión y se convierte un tipo de par/ ayudante de aula, puesto que asume la responsabilidad que le piden sus compañeros menores, haciéndolo con atención y buen trato y contando con la aprobación y el espacio del docente para que pueda realizar esta actividad adicional.

El cuadro 3 da cuenta de los procesos de enseñanza y aprendizaje observados, propiamente tales.

\subsection{Prácticas de enseñanza en la sala de clases}

En relación a las prácticas y particularidades de los procesos de implementación del currículum en las clases observadas, hubo dinámicas que contaron con mayor recurrencia. Los patrones de enseñanza que utilizaron los docentes fueron descritas en el apartado anterior, pudiendo diferenciarse entre aquellas que individualizan el trabajo en un estudiante o grupos de estos y los que organizaron la clase pensando en el grupo completo. En el primero de los casos, se identificaron dinámicas como dedicar períodos de minutos específicamente a cada grupo, tanto para enseñar, como para acompañar el trabajo, realizar actividades de aprendizaje diferenciadas o acompañar de manera diferenciada a los 
estudiantes en sus actividades, valiéndose en algunos casos de recursos, como una pizarra específica para el alumno.

En el segundo de los casos, se consideraron prácticas en que se solicitaba al grupo curso que trabajen, sin remarcar diferencias mayores entre grupos, Pero al mismo tiempo buscando alternativas de brindar acompañamiento particularizado. Como puede observarse, las prácticas de enseñanza están marcadas por el rol que asume el docente en el aula, ocurriendo en el primer patrón una mayor amplitud de opciones metodológicas implementadas. Por su parte, los dos casos se identificaron solo algunos episodios en que el docente organizaba un cierre de la clase en común con los diferentes estudiantes. En unos casos era dirigido por él o ella y en otros, le pedía a los estudiantes que presentaran o comentaran sobre determinado tema trabajado.

\subsection{Prácticas de monitoreo y evaluación}

Las prácticas de monitoreo y evaluación utilizadas dentro de la clase se enmarcaron también en los patrones que se han ido describiendo. En el caso del patrón de clases con actividades individualizadas o en grupo, el o la docente realizan visitas permanentes a cada mesa, para verificar el avance, resolver dudas y apoyar en el proceso de aprendizaje, siendo esta práctica la que más tiempo ocupa durante la clase.

Por su parte, en el caso de las clases que se organizaban con el grupo curso completo, las prácticas de monitoreo y evaluación ocurrieron principalmente a través de preguntas: al inicio de la clase para identificar el grado en que los estudiantes recuerdan lo que se está trabajando, en el desarrollo, para verificar si se entiende lo que se realizará o si se está avanzando conforme a lo solicitado y al cierre, para generar conclusiones sobre lo aprendido.

\subsection{Vínculos entre contenidos de la clase y el entorno o la vida cotidiana de los estudiantes}

Si bien en algunos casos se identifica de manera explícita este vínculo, en la mayor cantidad de los casos este aspecto no se observó con claridad. En uno de los casos en que sí ocurrió, se hizo un ejercicio de indagación científica referido a seres vivos en la observación de dos animales que viven en el río contiguo a la escuela. En otro caso, se estableció un vínculo entre una fábula con la vida en el campo y el ciclo del trabajo. Ese desafío pedagógico no fue completamente abordado en las clases observadas, situación que es llamativa debido al conjunto de especificidades que se ha observado que ocurren en este tipo de enseñanza. 
Cuadro 3. Descripción de clases observadas. Dimensión procesos de enseñanza y aprendizaje

\begin{tabular}{|c|c|c|c|c|c|c|c|c|c|c|c|c|c|c|c|}
\hline & & $\begin{array}{c}\text { ESC } \\
1 \\
\end{array}$ & $\begin{array}{c}\text { ESC } \\
2\end{array}$ & $\begin{array}{c}\text { ESC } \\
3\end{array}$ & $\begin{array}{c}\text { ESC } \\
4\end{array}$ & $\begin{array}{c}\text { ESC } \\
5\end{array}$ & $\begin{array}{c}\text { ESC } \\
6\end{array}$ & $\begin{array}{c}\text { ESC } \\
7\end{array}$ & $\begin{array}{c}\text { ESC } \\
8\end{array}$ & $\begin{array}{c}\text { ESC } \\
9\end{array}$ & $\begin{array}{c}\text { ESC } \\
10\end{array}$ & $\begin{array}{c}\text { ESC } \\
11\end{array}$ & $\begin{array}{c}\text { ESC } \\
12\end{array}$ & $\begin{array}{c}\text { ESC } \\
13\end{array}$ & $\begin{array}{c}\text { ESC } \\
14\end{array}$ \\
\hline \multirow{7}{*}{$\begin{array}{l}\text { Prácticas de enseñanza } \\
\text { y actividades } \\
\text { desarrolladas en la clase }\end{array}$} & $\begin{array}{l}\text { Explicación general de contenidos, } \\
\text { seguida por trabajo individual de } \\
\text { estudiantes con acompañamiento } \\
\text { individual. }\end{array}$ & $\mathrm{X}$ & $\mathrm{X}$ & $\mathrm{X}$ & & $\mathrm{X}$ & & $\mathrm{X}$ & $\mathrm{X}$ & $\mathrm{X}$ & & $\mathrm{X}$ & & $\mathrm{X}$ & \\
\hline & $\begin{array}{l}\text { Trabajo individual de estudiantes con } \\
\text { acompañamiento individual. }\end{array}$ & & & & $\mathrm{X}$ & & $\mathrm{X}$ & & & & $\mathrm{X}$ & & $\mathrm{X}$ & & \\
\hline & Cierre colectivo de la clase & & & & & $\mathrm{X}$ & $\mathrm{X}$ & $\mathrm{X}$ & $\mathrm{X}$ & & & & & & \\
\hline & Trabajo colectivo & & & & & & $\mathrm{X}$ & $\mathrm{X}$ & $\mathrm{X}$ & & & $\mathrm{X}$ & & & \\
\hline & $\begin{array}{l}\text { Trabajo de estudiantes organizados } \\
\text { por grado }\end{array}$ & & $\mathrm{X}$ & & & $\mathrm{X}$ & & & & & & & & & \\
\hline & Presentación de trabajo al curso & & & & $\mathrm{X}$ & & & & & & & & & & \\
\hline & $\begin{array}{l}\text { Modelamiento seguido de } \\
\text { acompañamiento individual }\end{array}$ & & & & & & & & & & & & & & $\mathrm{X}$ \\
\hline \multirow{5}{*}{$\begin{array}{l}\text { Prácticas de monitoreo } \\
\text { y evaluación }\end{array}$} & Monitoreo individual de actividades & $\mathrm{X}$ & $\mathrm{X}$ & & $\mathrm{X}$ & $\mathrm{X}$ & $\mathrm{X}$ & & & $\mathrm{X}$ & $\mathrm{X}$ & & $\mathrm{X}$ & $\mathrm{X}$ & \\
\hline & Monitoreo a todo el curso (colectivo) & & & $\mathrm{X}$ & & & & $\mathrm{X}$ & $\mathrm{X}$ & & & $\mathrm{X}$ & & & $\mathrm{X}$ \\
\hline & $\begin{array}{l}\text { Monitoreo por grupos/ niveles de } \\
\text { enseñanza }\end{array}$ & & $\mathrm{X}$ & & & & & $\mathrm{X}$ & $\mathrm{X}$ & & & & & & \\
\hline & Foco en estudiantes pequeños & & & & & & & & & & & & $\mathrm{X}$ & & \\
\hline & Foco en estudiantes con NEE & & & & & & & & & & & & & & $\mathrm{X}$ \\
\hline \multirow{3}{*}{$\begin{array}{l}\text { Vínculos entre } \\
\text { contenidos de la clase y } \\
\text { entorno/ vida cotidiana }\end{array}$} & Sin vinculaciones explícitas & & & & & & & $\mathrm{X}$ & & $\mathrm{X}$ & $\mathrm{X}$ & $\mathrm{X}$ & $\mathrm{X}$ & $\mathrm{X}$ & $\mathrm{X}$ \\
\hline & Vida rural/ entorno geográfico & & & $\mathrm{X}$ & & & $\mathrm{X}$ & & $\mathrm{X}$ & & & & & & \\
\hline & Edad de estudiantes & & $\mathrm{X}$ & & $\mathrm{X}$ & $\mathrm{X}$ & & & & & & & & & \\
\hline
\end{tabular}




\begin{tabular}{|c|c|c|c|c|c|c|c|c|c|c|c|c|c|c|c|}
\hline \multirow{9}{*}{ Recursos pedagógicos utilizados } & PowerPoint & $\mathrm{X}$ & & $\mathrm{X}$ & & & $\mathrm{X}$ & & $\mathrm{X}$ & $\mathrm{X}$ & & & & & \\
\hline & Guía de trabajo & $\mathrm{X}$ & & & & $\mathrm{X}$ & & $\mathrm{X}$ & $\mathrm{X}$ & $\mathrm{X}$ & $\mathrm{X}$ & & & $\mathrm{X}$ & \\
\hline & Pizarra & $\mathrm{X}$ & $\mathrm{X}$ & $\mathrm{X}$ & $\mathrm{X}$ & $\mathrm{X}$ & & $\mathrm{X}$ & & & $\mathrm{X}$ & $\mathrm{X}$ & & & \\
\hline & Textos escolares & & $\mathrm{X}$ & & $\mathrm{X}$ & $\mathrm{X}$ & & $\mathrm{X}$ & & & & & & $\mathrm{X}$ & \\
\hline & Material concreto & & & & & $\mathrm{X}$ & $\mathrm{X}$ & $\mathrm{X}$ & & $\mathrm{X}$ & & & $\mathrm{X}$ & & \\
\hline & Recursos naturales & & & & & & $\mathrm{X}$ & & & & & & & & \\
\hline & Útiles escolares & & & & & $\mathrm{X}$ & $\mathrm{X}$ & $\mathrm{X}$ & $\mathrm{X}$ & & & & $\mathrm{X}$ & $\mathrm{X}$ & \\
\hline & Otros textos & & & & & & & & & & & $\mathrm{X}$ & & & \\
\hline & Artículos deportivos & & & & & & & & & & & & & & $\mathrm{X}$ \\
\hline \multirow{3}{*}{ Clima de aula } & Tranquilo, respetuoso y trabajo fluido. & $\mathrm{X}$ & $\mathrm{X}$ & $\mathrm{X}$ & $\mathrm{X}$ & & $\mathrm{X}$ & & $\mathrm{X}$ & $\mathrm{X}$ & $\mathrm{X}$ & $\mathrm{X}$ & $\mathrm{X}$ & $\mathrm{X}$ & $\mathrm{X}$ \\
\hline & Medianamente tranquilo, con ruido, que dificulta la enseñanza. & & & & & $\mathrm{X}$ & & & & & & & & & \\
\hline & Tranquilo y respetuoso, pero con actitud pasiva de los estudiantes. & & & & & & & $\mathrm{X}$ & & & & & & & \\
\hline
\end{tabular}

Fuente: Elaboración propia. 


\subsection{Clima de aula}

En todas las clases observadas se identificó un clima caracterizado por su tranquilidad. Se observaron pocos episodios relevantes en que se interrumpió el trabajo planificado. Fueron clases en general silenciosas, con un niveles de conversación que no dificultaban las actividades planificadas. Además, se vislumbró una actitud de respeto y colaboración de parte de los estudiantes hacia los docentes, en el sentido que participan con interés en los procesos que son solicitados por el docente. Se observó a los estudiantes concentrados y trabajando permanentemente, lo que denota una actitud favorable a los procesos de aprendizaje. Sin embargo, predominó el trabajo individual por sobre el colectivo entre los estudiantes.

Por su parte, la relación entre docente y estudiantes se caracterizó por la cercanía y el cariño entre las partes. Se identificaron gestos y demostraciones de cariño y preocupación en ambos sentidos de la relación.

La descripción de las clases observadas evidenció un conjunto de desafíos relevantes para una docencia multigrado de calidad. Un primer elemento es la necesidad indiscutible de ofrecer oportunidades de aprendizaje a los diferentes estudiantes que participan de la clase, reconociendo sus diferencias. Este gran desafío tiene implicancias en el uso de prácticas pedagógicas y estrategias de monitoreo y evaluación, las que no siempre se evidenciaron con suficiente variedad. Asimismo, el bajo uso del contexto en este tipo de enseñanza parece ser una oportunidad no explotada en las clases observadas.

\subsection{Percepción de docentes sobre calidad de la enseñanza y necesidades de desarrollo profesional asociadas}

A continuación se presentará un análisis de las percepciones de los docentes participantes del estudio en relación con la calidad educativa en sus escuelas y los desafíos del desarrollo profesional docente. En primer lugar, se buscó identificar los principales desafíos que reconocen los docentes para su labor pedagógica, pensando en la calidad educativa, de manera de identificar puntos en común y diferencias con los procesos observados en las clases.

De manera transversal los diferentes entrevistados enfatizan que la enseñanza en estas escuelas prioriza la individualidad de los estudiantes, lo que se debe al número más reducido de alumnos, y la cultura más comunitaria de la escuela. A juicio de los entrevistados, el docente puede realizar prácticas más centradas en las características personales de sus alumnos y también tiene oportunidad de acompañar y monitorear sus avances y dificultades.

La educación que nosotros entregamos es personalizada, yo tengo once alumnos. En
el día yo puedo estar con todos (...) diez minutos con uno, con el otro, está el tiempo
para eso, entonces, es una educación personalizada. (Docente encargada de escuela)

El atributo recién mencionado impacta en la lógica general de los procesos de enseñanza y aprendizaje, lo que hace que los docentes prioricen los dos diseños para las clases que se desarrollan en estas escuelas, similares a los identificados en la observación de clases. El primer diseño de clases corresponde a organizar una clase considerando la globalidad de los estudiantes que están en la sala, quienes en su gran mayoría, están cursando diferentes niveles de enseñanza.

El segundo diseño de enseñanza, más frecuentemente mencionado por los entrevistados, consiste en organizar la clase definiendo desde el inicio diferencias entre los distintos 
estudiantes, referidas principalmente a que cursan distintos niveles de enseñanza. El docente explica los contenidos a un nivel de enseñanza y les indica una actividad, luego a otro nivel y así sucesivamente hasta pasar por todos los niveles. Una vez realizada la explicación a todos los niveles e indicadas las actividades respectivas, el docente atiende las dudas particulares de los estudiantes.

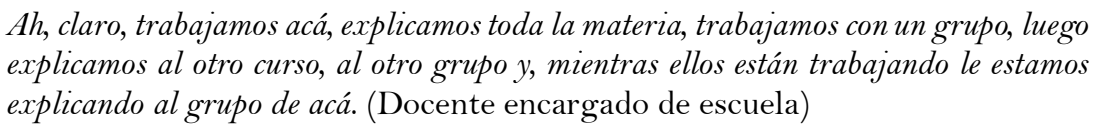

Algunas de las razones que los entrevistados indicaron respecto a la utilización de este segundo diseño de clase son las siguientes, las que aluden a la necesidad de asegurar cierto tipo de logros de aprendizaje, con independencia de las diferencias entre los estudiantes:

- Mientras los estudiantes de sexto básico deben prepararse para egresar a otra escuela, los que cursan primero básico deben aprender a leer. Esto afecta no solo a los contenidos de enseñanza, sino a las metodologías de enseñanza.

- Hay objetivos o ámbitos de aprendizaje en que no se considera factibles de trabajar en conjunto con los estudiantes que están en la clase.

- Se requiere "cubrir el currículum", o los objetivos de aprendizaje de todos los niveles, considerando la variedad de estudiantes que presentes y el tiempo disponible para la clase.

Para ambos diseños de clase los docentes realizan un análisis respecto de la estrategia que aseguraría de mejor forma la igualdad de condiciones para aprender, considerando las determinaciones y constreñimientos en que se desempeña la escuela. De esta forma, los docentes convergieron en la idea de que la definición del diseño de la clase es el mayor desafío referido a los procesos de enseñanza.

Nos dificulta un poco en que hacer clases, yo tengo que explicarle cosas a los de sexto.
Por ejemplo, si le estoy pasando fracciones y los chiquititos me están pasando números,
entonces, eso me dificulta un poco a hacer una retroalimentación a todo el curso, no
puedo hacerlo, tengo que separar porque tengo que retroalimentar. (Docente
encargado de escuela)

Dentro del marco de los diseños mencionados, las metodologías de enseñanza tienen importante grado de variación. Sin embargo, en todas las que mencionaron los docentes se buscaba organizar un proceso que respondiera a la individualidad de los requerimientos de los estudiantes y a cubrir los objetivos de aprendizaje de la asignatura. En esta lógica, el profesor o profesora asume un rol en que destina más tiempo al acompañamiento individualizado a los estudiantes, que a la impartición de enseñanza frente a todo el grupo curso.

Bueno, cuando estoy en matemática, la numeración la puedo trabajar con todos pero con distinta dificultad porque el $1^{\circ}$ año va a tener una dificultad distinta al de $2^{\circ}$ año que está trabajando en un ámbito numérico diferente.

Le voy a dar las instrucciones al $2^{\circ}$ año, al $3^{\circ}$, al $4^{\circ}$, me apoyo con los libros que da el ministerio, con guías que yo saco les tengo preparadas. (Docente encargado de escuela)

De los comentarios hechos por los docentes se observa que, en ocasiones, los recursos de enseñanza se convierten en el principal insumo para la clase. Esto ocurre, por ejemplo, en asignaturas en que el docente asume que tiene un menor dominio -particularmente inglés, música o educación física-, o cuando el docente ha identificado que le aportan a su 
enseñanza individualizada. Paradójicamente, pese a la relevancia que parecen adquirir los recursos pedagógicos para la enseñanza en este tipo de establecimientos, la baja disponibilidad de estos en las escuelas es una de las críticas sobre lo que hay mayor consenso.

Pero el Estado da subvención por cada alumno. Mira, llegó una cantidad de plata, pero si tengo 14 alumnos no puedo, no tengo los recursos como para comprar un laboratorio [de ciencias], aunque sea el más baratito. No sé, me gustaría tener internet, unas mesas, unas sillas de colores, pintar la escuela, pero con los recursos que me llegan... (Docente encargado de escuela)

Respecto al rol que tienen de los estudiantes en la clase, en los discursos prima la idea que se otorga una mayor cantidad de tiempo a las actividades de aprendizaje, ya sean individuales o grupales, autónomas o asistidas por el profesor. De acuerdo a una parte de los entrevistados, los docentes son requeridos por sus estudiantes para resolver inquietudes o ser guiados en su proceso de aprendizaje. Es en este marco, por ejemplo, puede existir, el rol del estudiante tutor.

Por ejemplo, yo tengo niños más avanzados. Me dedico al más débil; al otro, lo dejo
trabajando en algo que él pueda solo y le preguntan al compañero (...) A veces cuando
yo estoy haciendo algún trabajo de encargado del colegio, me vienen a preguntar y
digo "Maryori explícale tú un ratito", entonces ella tiene harta paciencia y es bien
habilosa. (Docente encargado de escuela)

Un aspecto adicional que se enfatiza en el discurso de los entrevistados es el fenómeno que ocurre con la llegada de los estudiantes a la escuela a cursar primero básico, lo que frecuentemente significa la primera experiencia de escolarización. Esta situación demanda una necesidad de especial consideración y preocupación de parte del docente, de manera de favorecer la inserción y nivelación del estudiante, en su primer año de enseñanza.

Cuando un estudiante llega la escuela, llega a $1^{\circ}$ básico. Lo que un niño aprendió en kínder que fue en un año, pero tú tienes que enseñarlo en un mes, o dos, eso es como un desafio". (Docente encargado de escuela)

El discurso de los docentes entrevistados reafirma la identificación de los patrones descritos en el proceso de observación y agrega elementos que permiten comprender mejor las definiciones de enseñanza. Como ha sido posible de observar, los docentes consideran la necesidad de equilibrar los desafíos propios de una enseñanza particularizada con las exigencias por las que deben responder como encargados de escuelas. Las estrategias de enseñanza se ponen así a prueba en momentos relevantes de la trayectoria escolar, al inicio de ésta y al terminarla.

A continuación se abordará la identificación de atributos profesionales que los docentes indican que debe contar un docente que se desempeña en aulas multigrado. Los entrevistados reconocieron dos grandes tipos de atributos relacionados con competencias para la enseñanza en aulas multigrado. El primer tipo de carácter personal, tuvo mayor cantidad menciones de parte de los entrevistados y alude a la percepción que para desempeñarse en una escuela pequeña, debe existir un interés por este tipo de educación, un alto compromiso y afecto por la escuela y los estudiantes, considerando condiciones precarias en términos de infraestructura y recursos.

Entonces yo creo que lo primero que todo, un compromiso cien por ciento del profesor, del equipo de trabajo y tratar de que los pocos recursos que llegan a nuestra escuela, en el sentido pedagógico, se utilicen de la mejor forma. No salir de la sala sin preguntarles a los niños si entendieron algo. To de repente juego con ellos que a mí se 
me olvidó algo y esto, pero es una forma más que nada para saber si lo que yo enseñé se les quedó en su cabecita. (Docente encargada de escuela)

El segundo tipo de atributos corresponde a competencias técnicas, en que se destacan las capacidades de adaptabilidad y flexibilidad para la generación de adecuaciones y adaptaciones curriculares. Las características de los alumnos, pero también las determinaciones que tiene la enseñanza en la escuela, obligan a los docentes a reflexionar sobre cómo adecuar el currículum, para responder a la individualidad de sus estudiantes, considerando los diferentes niveles de enseñanza que están coexistiendo en la sala y a las características del entorno o el contexto en que se encuentra emplazada la escuela.

\begin{abstract}
Hay que aprovechar el conocimiento que ellos tienen de su entorno, contextualizar lo que tú le enseñas dentro de lo sala de clases, para qué le va a servir. Porque, de repente los contenidos que vienen en los textos cierto hablan sobre realidades que son muy diferentes (Docente encargado de escuela)
\end{abstract}

Asimismo, se menciona que se debe contar también con una sólida formación profesional, que permita tener conocimientos de las diferentes asignaturas que se imparten y de los objetivos clave que deben priorizarse. Lo anterior también se vincula con la necesidad de manejar estrategias didácticas específicas para enseñar las diferentes asignaturas del currículum y metodologías que se adecuen a las características de sus estudiantes.

\title{
4.10. Percepciones respecto del desarrollo profesional docente en estos contextos
}

Los entrevistados concuerdan en que existen serias dificultades para poder participar de instancias de desarrollo profesional. El principal sería que no disponen de tiempo, debido a que desempeñan varios roles al interior de la institución. En segundo lugar, se destacan dificultades de acceso, debido a que se desempeñan en lugares alejados o que cuentan con problemas para desarrollarlo de forma virtual, por las dificultades que tienen con la conexión a internet. En este contexto, los participantes optan en muchos casos por la autoformación.

\footnotetext{
Yo creo que es la capacitación permanente, hay que estar capacitándose permanentemente para estar preparados para enfrentar un curso así, una clase, y no quedarnos atrás de los otros profesores de Chile, que siempre están en talleres. A nosotros nos cuesta un poco, porque somos rurales, para ir a un taller hay que ir a Puerto Montt a 70 kilómetros, un poco más yo creo, entonces, igual hay un esfuerzo de nuestra preparación. (Docente encargado de escuela)
}

Se consultó a los participantes sobre contenidos u objetivos relevantes de ser considerados en el desarrollo profesional en este contexto. Sin que se identifique un aspecto que congregue un mayor consenso, se mencionaron temas como estrategias concretas de enseñanza en aulas multigradas, planificación diversificada, uso de tecnologías, estrategias para enseñanza aprendizaje de estudiantes con necesidades educativas especiales o sobre cómo utilizar los materiales que llegan a la escuela.

\section{Discusión}

$\mathrm{Al}$ inicio de este trabajo, se presentaban tres preguntas claves que Coll (2009) relaciona con calidad educativa: cuáles son los objetivos y finalidades de la educación; qué características debiera tener el currículum escolar para responder a las necesidades de los estudiantes y la sociedad y de qué forma los sistemas educativos responden a exigencias educativas y necesidades de aprendizaje cada vez más diversas. Si se aplican estas 
preguntas al contexto de las aulas multigrado, los resultados de este estudio pueden aportar en la identificación de claves para la calidad educativa en ellas.

En relación con las preguntas sobre los objetivos y finalidades de la educación y las preguntas que debe tener el currículum, los resultados dan cuenta de la necesidad de equilibrar dos desafíos fundamentales: a) favorecer una enseñanza individualizada, que responda a las particularidades de los diferentes aprendizajes que requieren estudiantes de diferente grado y b) organizar una clase que garantice igualdad de condiciones para el aprendizaje de los estudiantes de distintos niveles, definir objetivos de aprendizaje que sean viables de trabajar con el conjunto de la clase, cubrir el currículum o los objetivos de aprendizaje para todos los niveles u optimizar el tiempo disponible durante la clase.

Ciertamente, estos objetivos se enfrentan desde un escenario marcado por la escasez de recursos económicos, una deficiente infraestructura pedagógica y casi nulo acceso a internet, como dificultades estructurales, y trato cercano y afectivo entre docentes y estudiantes, como activos o fortalezas.

Lo anterior se vincula con la pregunta sobre las respuestas que ofrece el sistema educativo, donde se identificaron importantes deudas. En este contexto, el rol del docente encargado de la escuela adquiere una relevancia que, en términos relativos, es mayor al de las escuelas más grandes, puesto que todo lo que ocurre en su interior depende en gran medida de los que éste haga. Ciertamente, la multiplicidad de roles que debe ejercer el director o docente encargado es un factor que pone en tensión la labor pedagógica. Este cargo se ve constreñido por la precariedad de las condiciones de trabajo en que estos actores se desempeñan: al haber pocos recursos económicos y humanos, las escuelas pequeñas no tienen la capacidad de contratar personal y los docentes encargados deben hacerse cargo de llevar a cabo diferentes tareas.

La existencia de un docente a cargo de toda la escuela implica necesariamente la ausencia o baja disponibilidad de horas de docentes especialistas que impartan asignaturas tales como inglés, músicas, artes visuales o musicales y educación física. En algunos casos, estos profesores no disponen de suficientes competencias o especialidad para enseñar esas áreas del conocimiento o no disponen de recursos pedagógicos de apoyo a la enseñanza.

En Chile, salvo casos aislados, no existe formación inicial o continua docente destinada a preparar a profesionales de la educación para trabajar en escuelas multigrado. El estudio permitió constatar además que el desarrollo profesional se realiza principalmente en base a la experiencia y la autoformación. Esta situación, además de ser inequitativa, constituye un riesgo para la calidad educativa, ya que los docentes no tienen suficientes oportunidades de aprendizaje y desarrollo profesional adecuados a su propio contexto de trabajo.

En el marco descrito, el estudio permite identificar claves relacionadas con la calidad de una clase multigrado, las que se asocian a requerimientos específicos de desarrollo profesional docente. Estas claves hacen sentido si se vinculan además con el paradigma de educación inclusiva, debido a la convergencia que existe entre ésta y los desafíos de la educación multigrado.

Desde esta perspectiva, las claves que se mencionarán a continuación consideran como visión el enfoque "de los apoyos", en que los profesionales de la educación desarrollan y despliegan habilidades para brindar sistémicamente los soportes que requiere cada estudiante (Sánchez- Gómez y López, 2020). Asimismo, desde la educación inclusiva se 
apunta al desarrollo profesional que permita una adecuada gestión de la educación diferenciada, lo que implica diseñar e implementar procesos de aprendizaje que consideren integralmente aspectos como un ambiente acogedor y seguro, la participación en el currículum o la evaluación continua de necesidades y avances de alumnos y alumnas (Glatzel, 2017).

Otro aspecto indispensable para la identificación de las claves es el aporte de la docencia al despliegue de la autodeterminación, para que los estudiantes desarrollen la autonomía que les permita ser gestores de su propia vida, y por lo tanto de su proceso de aprendizaje (Ramírez, 2019). Este aspecto, además de ser un fin en cualquier contexto, tiene implicancias fundamentales en las aulas multigrado, dadas las condiciones en las que se desempeñan.

A juicio de Duk, Cisternas y Ramos (2019), se trata entonces de favorecer un desarrollo profesional docente para la educación inclusiva, que favorezca cuatro dimensiones fundamentales: respetar la diversidad de todos los estudiantes, favorecer a todos los estudiantes, con altas expectativas, trabajar colaborativamente y aprender permanentemente sobre para enfrentar los desafíos de la enseñanza.

Un aspecto central para el despliegue de estas dimensiones es la reflexión sobre la propia práctica. Navarro (2017) indica que la educación inclusiva requiere de docentes que diseñen experiencias de aprendizaje considerando las experiencias y conocimientos previos de sus estudiantes.

Teniendo en cuenta estas definiciones fundamentales desde la educación inclusiva, se presentan a continuación las claves para la calidad en aulas multigrado que tienen implicancias en el desarrollo profesional en dicho contexto. Un primer aspecto alude a la planificación de clases. Más que seguir un formato tradicional, en este caso se esperaría que el docente cuente con ciertos diseños de clase según área del conocimiento, que pueda aplicar en su trabajo cotidiano y que cuenten con especificidades por grupos de estudiantes, según el grado que estén cursando. Dentro de estos diseños diferenciados, los grupos extremos en cuanto al grado (los que están ingresando y los que están saliendo) tendrán una particular necesidad de ser considerados.

En segundo lugar, la optimización del uso del tiempo de clases y la necesidad de implementación del currículum aparecen como un permanente punto de tensión para la calidad de la enseñanza. Esto está estrechamente relacionado con el diseño de las clases y apunta a generar un repertorio de trabajo que tenga cierta estabilidad en el quehacer docente, de forma de dar menor pie a la improvisación.

Vinculado a lo anterior, emerge la necesidad de desarrollar de conocimientos y habilidades para hacer adecuaciones curriculares. Esta tarea, para la que en los últimos años se ha desarrollado conocimiento y estrategias en el marco de la implementación de programas de inclusión, será fundamental para sostener el esfuerzo de trabajar con temas similares en diferentes grados de enseñanza.

Desde el plano de los procesos de enseñanza, se identificó que se privilegia el trabajo individual y que este proceso se funde con el de monitoreo de los aprendizajes. Dada la centralidad de este proceso, se vuelve relevante consolidar ciertas rutinas y prácticas de enseñanza individualizada y a ello agregar otras experiencias. Ciertamente, la enseñanza personalizada (Ferrini, 2000) como método puede ser un aporte a los estudiantes de las escuelas multigrado, debido al énfasis que otorga al estudiante en la planificación y 
ejecución de su propio aprendizaje. Esto puede convertirse además en un apoyo al docente en el uso de su tiempo.

Siguiendo con lo anterior, el desarrollo de competencias para monitorear el aprendizaje de estudiantes en diferente grado dentro de una misma clase, es otra de las claves identificadas para la calidad educativa en este contexto. La perspectiva de la evaluación para el aprendizaje (Mineduc, 2006) puede ser un aporte al desarrollo profesional en este objetivo, considerando la posibilidad de definir previamente criterios de evaluación que al mismo tiempo se conviertan en proyecciones para el aprendizaje de los estudiantes. Es decir, puede convertirse en una herramienta de monitoreo y de enseñanza, al mismo tiempo.

Junto a todo lo anterior, se identificaron experiencias que dieron cuenta del aporte del "efecto par" en el aprendizaje en estos contextos. Sin embargo, no fue una práctica recurrente y en oportunidades se evidenció que se hacía de forma espontánea. Si bien ya se conocen los múltiples beneficios del efecto par en cualquier contexto de aprendizaje, en el caso de las escuelas multigrado se convierten en una espacial oportunidad, debido al endémico problema de tiempo disponible para la enseñanza. En esta línea, la formación para la generación de estrategias e interacciones de aprendizaje entre pares se presenta como un ámbito que puede ser de gran apoyo para la calidad.

Otro de los aspectos que no se distinguió con claridad en las observaciones ni las entrevistas, dice relación con el cierre de la clase. Tal como en otros contextos, ese momento en muchos casos se obvia, "porque no alcanza el tiempo". En el caso puntual de la enseñanza multigrado, el cierre es un espacio tan relevante como dificultoso, debido a la diversidad de alumnos en el aula y a que se ha privilegiado la enseñanza individualizada en la clase, lo que minimiza los espacios de socialización de los aprendizajes. En línea con lo anterior, los docentes podrían desarrollar estrategias específicas que ayuden a que el momento del cierre sea un espacio que permita ese proceso.

Pudieron observarse con claridad las limitaciones y riesgos de abordar todo el currículum desde una perspectiva generalista, que además se implementa en un contexto sociocultural diferente a la educación regular. En este ámbito, los recursos de aprendizaje de diferente tipo se pueden convertir en apoyos importantes para cubrir estas carencias, pero si no se identifican y utilizan adecuadamente, pueden incluso ser contraproducentes para el aprendizaje. Por esta razón, parece relevante generar instancias de desarrollo profesional que permitan desarrollar la competencia para identificar y seleccionar recursos actualizados y de calidad, así como también para socializar prácticas exitosas al respecto.

El clima de aula se consolidó como uno de los activos más permanentes en este tipo de aula. Sin embargo, la naturaleza de la clase individualizada no permite potenciarlo, o ponerlo a prueba, debido a la baja interacción que existe entre los estudiantes. Flecha (2009) indica que las interacciones dialógicas no solo tienen efecto en dar voz a todos los estudiantes, sino que también mejoran las prácticas educativas, avanzando hacia estadios más avanzados que la mera pretensión de entenderse con los estudiantes.

Uno de los grandes problemas para avanzar en los aspectos que se han identificado es la soledad en la que se desempeñan los docentes. Sin equipos estables de trabajo, utilizan todo su tiempo de trabajo en labores de enseñanza, gestión y administración de su propia escuela, a kilómetros de distancia de su más cercano colega. Por esta razón, un factor fundamental para un desarrollo profesional docente que favorezca la calidad de los aprendizajes será la generación de instancias de encuentro profesional entre pares. Así 
como en el caso de la labor individual del docente, las reuniones de docentes multigrado podrían planificarse para trabajar aspectos como los mencionados e innovar en formas de acompañamiento entre pares, utilizando prácticas como la visita de un colega o aprovechando las oportunidades que abre el uso de tecnologías. Así, por ejemplo, un docente podría grabar su clase con un celular y compartirlo con sus colegas, de manera de aportar a la discusión colectiva sobre sus propias prácticas.

\section{Agradecimientos}

Los resultados presentados son productos de un estudio diseñado y financiado por la Agencia de Calidad de la Educación a través de la Licitación Pública ID 72 1703-30-LP18. Todo el material producido en el marco de la investigación es propiedad de la Agencia de Calidad de la Educación.

Los datos utilizados para escribir este artículo fueron levantados en el contexto de un estudio realizado por la Agencia de Calidad de la Educación, cuyo objetivo fue establecer una definición de calidad de la educación escolar, a través de la identificación de atributos clave que definen una educación de calidad en establecimientos educacionales pequeños chilenos, con el propósito de aportar en la definición de la medición de calidad de dichos establecimientos. Así, el estudio de las clases en establecimientos multigrado es una parte del análisis de los mencionados atributos, sin haber sido el núcleo del estudio.

\section{Referencias}

Agencia Europea para el Desarrollo de la Educación del Alumnado con Necesidades Educativas Especiales. (2012). Formación del profesorado para la educación inclusiva. Perfil profesional del docente en la educación inclusiva. Agencia Europea para el Desarrollo de la Educación del Alumnado con Necesidades Educativas Especiales.

Ainscow, M., Booth, T. y Dyson, A. (2006). Mejorando escuelas, desarrollando inclusión. Routledge.

Arnaiz, P. A., Escarbajal, A. y Caballero, C. M. (2017). El impacto del contexto escolar en la inclusión educativa. Revista de Educación Inclusiva, 10(2), 195-210.

Ávila, B. R. (2017). Experiencias pedagógicas significativas de educación rural en Colombia, Brasil y México. Revista del Centro de Investigación, 14(48), 121-158. https://doi.org/10.26457/recein.v12i48.1486

Boix, R. (2014). La escuela rural en la dimensiónn territorial. Innovación Educativa, 24, 89-97.

Boix, R. y Bustos, A. (2014). Una aproximación a las actividades escolares y los recursos didácticos desde la perspectiva del profesorado. Revista Iberoamericana de Evaluación Educativa, 7, 2943.

Bustos, A. (2010). Aproximación a las aulas de escuela rural: Heterogeneidad y aprendizaje en los grupos multigrado. Revista de Educación, 352, 353-378.

Centre for Education Statistics and Evaluation. (2013). Rural and remote education: Literature review. New South Wales Office of Education.

Clarke, S. y Stevens, E. (2009). Sustainable leadership in small rural schools: Selected Australian vignettes. Journal of Educational Change, 10, 277- 293. https://doi.org/10.1007/s10833008-9076-8 
Coll, C. (2009). Enseñar y aprender en el siglo XXI. el sentido de los aprendizajes escolares. En A. Marchessi, J. Tedesco y C. Coll (Eds.), Calidad, equidad y reformas en la enseñanza (pp. 101112). Santillana.

Creswell, J. W. y Plano, V. L. (2007). Designing and conducting mixed methods research. Sage.

Downes, N. y Roberts, P. (2016). Revisiting the schoolhouse: A literature review on staffing rural, remote and isolated schools in Australia 2004-2016. Australian and International Journal of Rural Education, 7, 1- 24.

Duk, C., Cisternas, T. y Ramos, L. (2019). formación docente desde un enfoque inclusivo. A 25 años de la Declaración de Salamanca, nuevos y viejos desafíos. Revista Latinoamericana de Educación Inclusiva, 13(2), 91-109. https://doi.org/10.4067/S0718-73782019000200091

Ferrini, R. (2000). Hacia una educación personalizada: Una alternativa en la educación. Limusa.

Flecha, R. (2009) Cambio, inclusión y calidad en las comunidades de aprendizaje. Cultura y Educación, 21(2), 157-169. https://doi.org/10.1174/113564009788345835

Fullan, M. (2005). Leadership \& sustainability: System thinkers in action. Corwin Press.

Galaz, C. (2018). Educación de calidad: Política pública y percepciones de docentes de escuelas rurales multigrado (Tesis de doctorado). Universidad Alberto Hurtado, Chile.

Glatzel, G. A. (2017). Clases diversas en las escuelas de Estados Unidos. La importancia de una educación inclusiva. Revista de Educación Inclusiva, 10(2), 79-98.

Godoy, F., Varas, L., Martínez, N., Treviño, E. y Meyer, A. (2016). Interacciones pedagógicas y percepción de los estudiantes en escuelas chilenas que mejoran: Una aproximación exploratoria. Estudios Pedagógicos, 42(2), art 8. https://doi.org/10.4067/So718-07052016000400008

Hopkins, D. y Reynolds, D. (2002). The past, present and future of school improvement. DfES.

Martinic, S. y Vergara, C. (2007). Gestión del tiempo e interacción del profesor-alumno en la sala de clases de establecimientos de jornada escolar completa en Chile. REICE. Revista Iberoamericana sobre Calidad, Eficacia y Cambio en Educación, 5(5), 3- 20.

Mineduc. (2006). Evaluación para el aprendizaje. Enfoque y materiales prácticos para lograr que sus estudiantes aprendan más y mejor. http://www.rmm.cl/sites/default/files/usuarios/yvivanco/File/EVALUACION\%2OPA RA\%20EL\%20APRENDIZAJE.pdf

Mourshed, M., Chijioke, C. y Barber, M. (2010). How the world's most improved school systems keep getting better. En M. Mourshed, C. Chijioke y M. Barber (Eds.) How the world's most improved school systems keep getting better (pp. 135-158). McKinsey and Company.

Navarro, M. (2017). Mejorar la calidad de la educación inclusiva: Propuesta de indicadores. Revista de Pedagogía, 38(102),122-143.

OECD. (2010). OECD rural policy reviews Québec. OECD.

Panizzon, D. (2011). Teaching secondary science in rural and remote schools: Exploring the critical role of a professional learning community. En D. Corrigan y J. Dillon (Eds.), The professional knowledge base of science teaching (pp. 173-188). Springer. https://doi.org/10.1007/978-90-481-3927-9_10

Ramírez A. R.(2019). La autodeterminación como medio promotor del desarrollo humano a partir de la diversidad de los contextos educativos. Revista de Educación Inclusiva, 12(2), 152-170.

Strauss, A. L. y Corbin, J. (2002). Bases de la investigación cualitativa: Técnicas y procedimientos para desarrollar la teoría fundada. Editorial Universidad de Antioquia. 
Tiana, A. (2009). Calidad, evaluación y estándares: algunas lecciones de las reformas recientes. En A. Marchessi, J. Tedesco, C. Coll (Eds.), Calidad, equidad y reformas en la enseñanza (pp. 5797). Santillana.

UNESCO. (1989). Multigrade teaching in single teacher primary school. UNESCO.

Valenzuela, J. P., Bellei, C., Sevilla, A. y Osses, A. (2009). ¿Qué explica las diferencias de resultados PISA Matemática entre Chile y algunos países de la OCDE y América Latina? En L. Cariola, G. Cares y E. Lagos (Eds.), ¿¿Qué nos dice PISA sobre la educación de los jóvenes en Chile? Nuevos análisis y perspectivas sobre los resultados en PISA 2006 (pp. 105-148). Ministerio de Educación, Unidad de Curriculum y Evaluación.

Wildy, H. y Clarke, S. (2011). Innovative strategies for small and remote schools. A literature review. The University of Western Australia.

\section{Breve CV del autor}

\section{Luis Felipe de la Vega}

Sociólogo, magíster en Política y Gobierno, magíster en Ciencias de la Educación, doctor en Ciencias de la Educación. Actualmente se desempeña en la Escuela de Investigación y Postgrado de la Universidad Cardenal Silva Henríquez. Sus objetos de investigación son el mejoramiento educativo, el desarrollo profesional docente y las políticas educacionales. Se ha desempeñado como investigador, consultor $\mathrm{y}$ evaluador de programas gubernamentales. Ha prestado acompañamiento y asesoría a establecimientos educacionales e instituciones públicas. ORCID ID: https://orcid.org/0000-0002-46106359. Email: luis.delavega@ucsh.cl 